\title{
The weighting of product design specification for a composite side-door impact beam using the analytic hierarchy process method
}

\begin{abstract}
In this study, the analytic hierarchy process (AHP) method is employed to determine the weightage criteria for product design specification (PDS) of a side-door impact beam. Six criteria for PDS have been chosen to be judged using the AHP pairwise comparison technique by ten decision makers from an automotive engineering and product design background. The results show that performance has the highest weightage, which is 0.3566 , follows by product cost and weight with the values of 0.1971 and 0.1690 , respectively. Environment and disposal are at fourth (0.1127) and fifth (0.0857) places, respectively, whilst size generates the lowest weightage (0.0789). The AHP consistency ratio (CR) also shows the obtained average CR value is below 0.1 , which proves that the decision makers' judgement is consistently high. In conclusion, this study indicates that the utilisation of the AHP method can assist in systematically determining the weightage criteria in the MCDM method.
\end{abstract}

Keyword : Analytic hierarchy process; AHP; Multiple criteria decision making; MCDM; Sidedoor impact beam; Weightage criteria 